Cinémas

Revue d'études cinématographiques

Journal of Film Studies

\title{
Ciné-romans : le livre du film
}

\section{Jacqueline Viswanathan}

Volume 9, numéro 2-3, printemps 1999

Les Scénarios fictifs

URI : https://id.erudit.org/iderudit/024784ar

DOI : https://doi.org/10.7202/024784ar

Aller au sommaire du numéro

Éditeur(s)

Cinémas

\section{ISSN}

1181-6945 (imprimé)

1705-6500 (numérique)

Découvrir la revue

Citer cet article

Viswanathan, J. (1999). Ciné-romans : le livre du film. Cinémas, 9(2-3), 13-36. https://doi.org/10.7202/024784ar

\section{Résumé de l'article}

Cet article analyse certains textes d'Ingmar Bergman, de Louis Malle, d'Éric Rohmer et de François Truffaut qui, tout en étant proches du roman, sont aussi des scénarios qui ont été publiés après le tournage des films. Nous y proposons une lecture de ces ciné-romans qui met au jour l'étape de l'écriture du scénario comme exploration et mise en fiction cinématographique d'un passé personnel. Nous montrons également l'importance du sujet de renonciation. Cette lecture autre révèle des aspects qui enrichissent et diversifient la conception traditionnelle du scénario comme un texte à prédominance performative. 


\title{
Ciné-romans : le livre du film
}

\author{
Jacqueline Viswanathan
}

\begin{abstract}
RÉSUMÉ
Cet article analyse certains textes d'Ingmar Bergman, de Louis Malle, d'Éric Rohmer et de François Truffaut qui, tout en étant proches du roman, sont aussi des scénarios qui ont été publiés après le tournage des films. Nous y proposons une lecture de ces ciné-romans qui met au jour l'étape de l'écriture du scénario comme exploration et mise en fiction cinématographique d'un passé personnel. Nous montrons également l'importance du sujet de l'énonciation. Cette lecture autre révèle des aspects qui enrichissent et diversifient la conception traditionnelle du scénario comme un texte à prédominance performative.
\end{abstract}

\begin{abstract}
This article analyzes a number of screenplays by Ingmar Bergman, Louis Malle, Eric Rohmer and François Truffaut which, while being close to novels by certain features, are also shooting scripts. We propose a reading of these cine-novels which reveals the process of screenplaywriting as linked to the exploration of a personal past through a cinematic vision. We also underline the importance of the writing subject in these texts. Our reading reveals new aspects of the screenplay as genre, besides its traditional view as performative discourse.
\end{abstract}

Qu advient-il du scénario après le tournage du film? Presque toujours, il disparaît, enterré dans les archives des maisons de production. Par chance, il est parfois conservé dans des collections de cinémathèques ou de particuliers. Sa fonction le destine 
en effet à un groupe restreint de lecteurs pour qui il est l'épure d'un film à venir. Le même sort est réservé aux innombrables scénarios jamais tournés, condamnés, eux aussi, à dormir dans les cryptes de M.G.M. ou d'autres studios. Et pourtant, le scénario n'est pas toujours un texte évanescent, réservé aux pratiquants. Depuis l'avènement du cinéma, il s'est publié un très grand nombre de scripts s'adressant non seulement aux cinéphiles, mais aussi à des lecteurs non spécialistes. Le scénario transcende alors sa vocation première. Comme la pièce de théâtre, il survit à la représentation et peut faire l'objet d'une lecture autre où le monde fictif se construit comme un spectacle dans l'imagination du lecteur.

Il faut différencier, parmi les scénarios publiés, entre, d'une part, continuités dialoguées, découpages techniques et autres scénarios de tournage qui sont des documents de travail étroitement liés à la production du film et d'autre part, les scénarios modifiés en vue d'une publication, accompagnés de préfaces et de commentaires, se rapprochant ainsi davantage d'une ouvre littéraire autonome. Parmi ces scénarios élaborés, retravaillés ou fictifs, distinguons trois groupes principaux. Le premier comprend les romans-scénarios, romans écrits sous forme de scénarios évoquant des films imaginaires, comme Neige noire d'Hubert Aquin ou Histoire de cour de Jacques Savoie. Le deuxième inclut des textes très variés: ébauches de scénarios aux formes très libres, fantasmes de films rêvés par des écrivains. Les scénarios fictifs rassemblés par Christian Janicot dans l'Anthologie du cinéma invisible témoignent de la diversité et de la qualité poétique de ces textes. Enfin, le troisième groupe dont il s'agira dans cet article se compose de scénarios qui, à la différence des précédents, se lisent dans la perspective d'un film déjà réalisé.

Ces textes, nous les appellerons ciné-romans pour bien marquer ce qui les distingue des continuités dialoguées et découpages techniques publiés, par exemple, par L'Avant-Scène du Cinéma. Dès l'époque du muet, des éditeurs ont voulu profiter de l'énorme popularité de certains films et prolonger leur succès par la publication d'une version romancée du scénario. Parmi les plus connus, on compte Fantômas de Louis Feuillade (1913), 
Les Mystères de New York de Louis Gasnier (1915'). Dès le début du siècle, des maisons telles que Gallimard et Hachette ont lancé des collections consacrées à ce type d'écrits. Bien que ces premiers ciné-romans n'aient pas toujours connu le succès escompté, la publication d'adaptations romancées de scénarios se poursuit jusqu'à nos jours. Elles représentent donc, en tentant de traduire les images du film en mots, le parcours inverse de l'adaptation du roman en film. Certains auteurs de ciné-romans ont essayé de créer un mode d'écriture nouveau qui refléterait les particularités de ce média en préservant certains aspects du découpage: texte morcelé, absence du narrateur, omniprésence du narrataire, style elliptique et dépouillé. Ces tentatives, pour intéressantes qu'elles soient, restent rares. Dans les novellisations contemporaines qui continuent à rencontrer un certain succès, on note au contraire une démarche constante qui consiste à effacer, dans le livre du film, toute trace d'une écriture cinématographique. Car il s'agit de donner au lecteur la possibilité de retrouver le plaisir qu'il avait ressenti, en recréant pour lui un monde imaginaire dont l'histoire et les personnages sont ceux du film qu'il a aimé. Ainsi, par exemple, la collection "Pocket Cinéma ${ }^{2}$ " réunit, sans les distinguer, des romans qui connaissent un renouveau de succès grâce à leur adaptation cinématographique, des versions romancées de scénarios (tel Philadelphia, "un roman de Christopher Davis, d'après un scénario de Ron Nyswaner») et des romans écrits a posteriori, à partir du film. Les adaptations cinématographiques de grands classiques donnent parfois lieu à de curieux phénomènes de transmédialité: le roman de Charles Dickens, Great Expectations suscite une novellisation, Great Expectations de Deborah Chiel (1998), "à partir du film tiré du roman de Charles Dickens». Les auteurs de novellisations évitent d'adopter des procédés narratifs qui dépayseraient le lecteur. Ils dépassent le contenu de l'image, révèlent la psychologie des personnages pour permettre une identification affective. Ils ménagent des transitions assurant la cohésion narrative. Tout laisse croire qu'une rupture avec les conventions romanesques, une expérimentation avec une écriture qui se rapprocherait du scénario, pourrait détruire le plaisir du spectateur lecteur. 
Cependant, entre ces deux extrêmes, découpages techniques d'une part, adaptations romancées de l'autre, il existe des cinéromans qui, par leur forme, le travail d'écriture auquel ils ont été soumis, comportent un intérêt particulier, autre que documentaire, tout en présentant un type de discours original. Ils témoignent de la naissance d'un genre nouveau, issu d'une rencontre entre les images et les mots. Textes hybrides, ces cinćromans sont, à certains égards, proches du discours romanesque, mais tout en préservant des aspects propres à l'écriture cinématographique.

\section{Écrivains cinéastes}

Parmi les plus connus, dans le domaine français, on compte les ciné-romans d'Alain Robbe-Grillet et les «textes-théâtrefilms» de Marguerite Duras. Écrivains, avant de devenir cinéastes, Robbe-Grillet et Duras ont apporté à leur expérience de scénaristes une conscience aiguë du retentissement de l'image sur les conventions de l'écriture scénaristique. Leur rencontre avec le cinéma prolonge leur recherche de romanciers qui, en transformant les conventions et les procédés romanesques, détruisent l'illusion mimétique. Robbe-Grillet et Duras publient en effet leurs scénarios, modifiés en vue de publication. Plusieurs études critiques ont déjà montré l'originalité de ces cinéromans ${ }^{3}$. Ceux-ci poussent jusqu’à leurs limites certains aspects propres au scénario, les différenciant de l'écriture romanesque: la fragmentation du texte, son caractère elliptique qui l'ouvre à une multiplicité d'interprétations, sa disposition typographique. Leurs ciné-romans ne prétendent en aucune façon se substituer au film. Ils disent au contraire toute la distance qui sépare les images de l'écriture.

Robbe-Grillet incorpore dans ses textes toute une réflexion sur cette expérience particulière qui consiste à représenter un film par des mots. Loin de chercher à occulter les marques du média cinématographique, il inclut des directives techniques particulièrement abondantes qui contribuent à mettre en relief tous les aspects déroutants du point de vue de la caméra. Il bloque la visualisation au lieu de l'encourager et présente une réflexion sur le processus de lisibilité particulier au scénario qu'il 
dissocie ainsi délibérément du littéraire. Par ailleurs, il ne cesse de souligner la possibilité d'interprétations multiples à partir de ces descriptions forcément incomplètes et fragmentaires des images. Le ciné-roman se présente donc comme l'œuvre ouverte par excellence.

Marguerite Duras refuse de subordonner le scénario au film, l'écriture au cinéma. Dans l'introduction au script de Navire Night qu'elle a retravaillé en vue de sa publication, elle déclare: "Je pense que ces précautions je les ai prises pour tenter d'effacer les traces du film afin d'éviter que le lecteur passe par lui aux dépens de sa propre lecture" (p. 10). Cependant, ses cinéromans different radicalement des novellisations. Elle fait des lacunes du scénario une des forces de ce texte hybride dont elle exploite systématiquement le manque à voir. Marguerite Duras accepte cette contrainte pour la mettre au service d'un art de l'ellipse et du dépouillement. Elle adopte alors les caractéristiques, fonctionnelles à l'origine, du scénario pour en faire des procédés expressifs visant un certain mode de lecture. Une écriture blanche, serrée, minimaliste dit, comme chez RobbeGrillet, mais dans un style bien à elle, l'incertitude de la signification. Le vide entre le texte et l'image crée un espace virtuel, un univers opaque, imprécis, mais d'autant plus ouvert aux fantasmes et à l'imaginaire personnel. Marguerite Duras crée consciemment des tex.tes hybrides en deçà des catégories traditionnelles. Elle commente ainsi Détruire, dit-elle:

C'est un livre cassé du point de vue romanesque. Je crois qu'il n'y a plus de phrases. Et par ailleurs, il y a des indications scéniques qui rappellent les scripts: "soleil ", "septième jour ", "chaleur": ce sont des indications de scénarios d'habitude... c'est-à-dire que je voudrais qu'il y ait la matière à lire le plus décantée possible du style [...]. Il n'y avait pas d'idée de film, mais il y avait l'idée d'un livre, comment dire ça d'un livre qui pouvait être à la fois soit lu, soit joué, soit filmé (p. 45).

Chez Robbe-Grillet et Duras, il est évident que leur expérience préalable d'écrivain a fortement influencé leur rapport au scénario. Elle a joué dans leur prise de conscience du style qui 
lui est propre et donc des possibilités d'une écriture nouvelle. Tous deux ont marqué leurs textes de leur vision et de leur style profondément personnels. Les premiers ciné-romans de RobbeGrillet, les premiers textes hybrides de Marguerite Duras ont été publiés à la fin des années soixante. Il est possible que leur pratique originale et créative d'une écriture cinématographique ait inspiré ces romans parus au cours de la décennie suivante: Prochainement sur cet écran de Pierre Turgeon, Neige noire d'Hubert Aquin, The Last Words of Dutch Schulz de William Burroughs, Pilote de chasse de Kenneth Gangemi. La forme du scénario y sert de modèle à une écriture expérimentale qui renouvelle les procédés romanesques ${ }^{4}$. Robbe-Grillet et Duras ont aussi donné à ce type d'écrits une respectabilité littéraire dont se soucient peu les auteurs de novellisations. La critique, qui avait jusque-là complètement ignoré ce domaine, a reconnu tout l'intérêt et l'originalité de ces textes et leur a donné une place importante dans l'œuvre de ces écrivains. En effet, les ciné-romans d'Alain Robbe-Grillet et les textes hybrides de Marguerite Duras ont suscité davantage d'analyses et de commentaires que leurs films. Les scénarios d'écrivains réputés sont à présent publiés et reconnus comme des cuvres à part entière, représentant un aspect important de leur créativité.

\section{Cinéastes écrivains}

Il existe d'autres textes, différents des découpages techniques, souvent retravaillés en vue de publication, et qui méritent, eux aussi, une lecture autonome. Nous pensons en particulier à certains scénarios de réalisateurs connus: L'Argent de poche et Les Aventures d'Antoine Doinel de François Truffaut, Le Souffle au cour et Au revoir les enfants de Louis Malle, Six Contes moraux d'Éric Rohmer et Les Meilleures Intentions d'Ingmar Bergman. A cause du caractère autobiographique de ces films, ces scénarios occupent une place à part dans l'œuvre de ces cinéastes écrivains. Ils sont révélateurs d'un stade particulier de la création où s'exprime une expérience intime et personnelle que le film va extérioriser. Ils intègrent aussi une subjectivité, les marques d'une énonciation et une présence auctoriale dont l'absence est, par ailleurs, une des marques distinctives du scénario. Pénétrés 


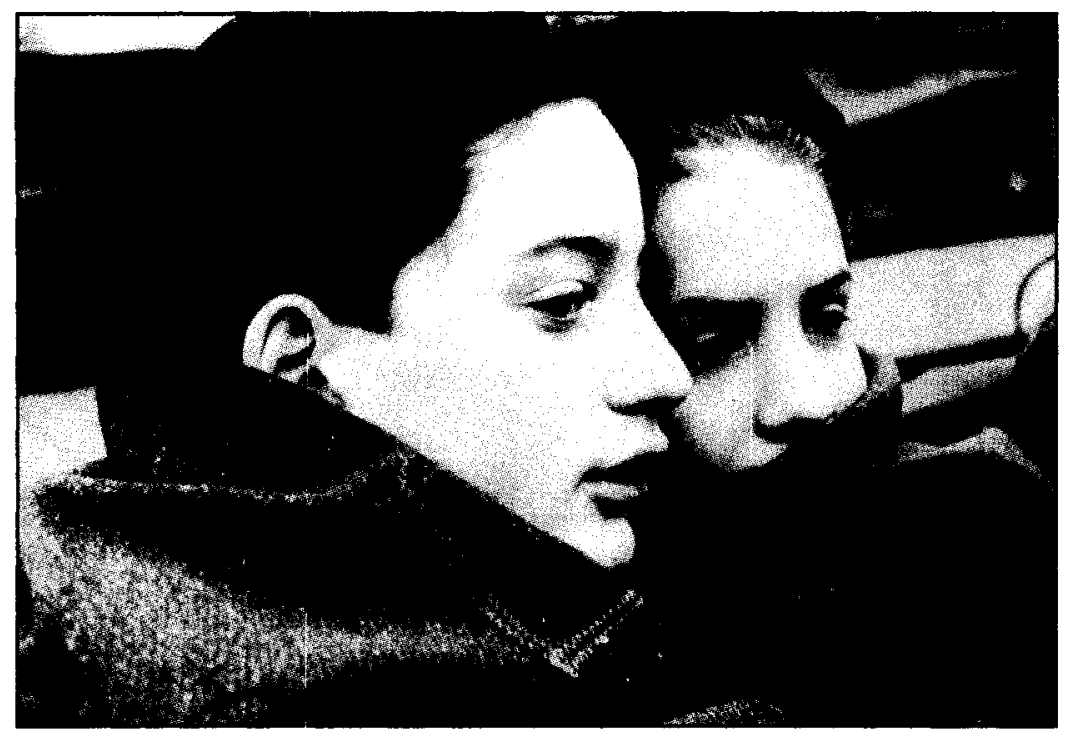

\section{Av revoir les enfants de Lovis Malle (1987)}

de littérature, Truffaut, Malle, Rohmer et Bergman ont créé des textes où ils n'ont pas craint d'user librement de divers procédés romanesques. Cinéastes écrivains, ils adoptent une pratique scripturale qui accomplit le parcours inverse des écrivains cinéastes. Ainsi les textes de Robbe-Grillet expriment-ils sa fascination pour l'appareillage cinématographique alors que les scénarios de Marguerite Duras usent savamment de l'écriture blanche et des ellipses qui démarquent le scénario du littéraire et du romanesque. Chez ces metteurs en scène qui adoptent spontanément une vision cinématographique, l'étape du scénario correspond à une écriture très libre, visant la création d'un monde imaginaire plutôt que les modalités spécifiques du tournage du film.

\section{Une autre lecture du scénario}

De prime abord, le scénario se conçoit comme un texte performatif, composé de directives pour la réalisation du film. Il s'adresse donc à des lecteurs spécifiques, chargés de leur exécution: metteur en scène, acteurs, techniciens. Comme chacun 
sait, les modalités du tournage et la production du film different considérablement du monde fictif, composé d'actions et de personnages que construira le spectateur. Cette lecture du scénario comme plan de travail prédomine; elle motive une conception du scénario comme texte fonctionnel, qui est celle des manuels, et en souligne tous les aspects non littéraires.

Les textes dont il s'agit dans cet article appellent une autre lecture du scénario, non moins importante, et qu'un article de Jean Alter permet de mieux mettre en lumière. Il y distingue deux façons de lire une pièce de théâtre. L'une est celle du metteur en scène, "[...] when the text is not read as fiction but as a step in the theatrical process, a performance to be. Such a reading will yield "in the mind's eye", what shall be called a virtual performance» (p. 49). Ainsi, le metteur en scène de théâtre imagine-t-il le rythme d'une scène ou le ton des acteurs. Pour sa part, le metteur en scène de cinéma pense au cadrage, à la sélection des plans... Cependant, ce stade d'élaboration du spectacle est précédé d'une autre lecture:

The virtual performance, when viewed as a referent of the text, always occurs after the play is read as literature... The formulation of meaning precedes the visualization of the performance. Where does it originate? It would seem that only one answer fits: the meaning must be drawn from the literary meaning. «I [the director] shall stage Vladimir [in Waiting for Godot] as a Von Sydow figure because I read him first as a pilgrim waiting for God " (p. 51).

Cette lecture que Jean Alter nomme, à défaut d'autre terme, littéraire se pratique spontanément pour les pièces de théâtre. En revanche, la conception dominante du scénario souligne son caractère performatif. Or, la lecture des ciné-romans de Malle, de Truffaut, de Rohmer et de Bergman permet de mettre en relief la création d'un monde qui, tout en étant inséparable d'une vision cinématographique, évoque des fictions profondément marquées par la personnalité de leurs auteurs, donnant au lecteur non spécialiste la possibilité d'imaginer des actions, des personnages, des lieux, une atmosphère, sous la forme d'un spectacle de l'esprit. Par ailleurs, cette lecture orientée vers 
l'imaginaire et sa signification plutôt que les modalités de la représentation est une notion clé pour l'analyse du processus d'intermédialité, car c'est à travers elle que se fait le passage d'un média à l'autre. Certes, ces ciné-romans sont exceptionnels par l'orientation littéraire de leurs auteurs et un mode d'écriture proche du romanesque, mais ils nous permettent de découvrir cette autre lecture suscitée par tout scénario.

\section{Louis Malle: un regard sur l'enfance}

Parmi les nombreux films réalisés par Louis Malle (dont les découpages techniques sont disponibles dans la collection L'Avant-Scène du Cinéma), trois scénarios occupent une place à part: Le Souffle au cœur, Au revoir les enfants et Milou en mai ont été publiés chez Gallimard sous la forme de ciné-romans. Ces textes ne contiennent en effet aucune indication technique. Cependant, ils different manifestement des novellisations par le mode scénique du discours qui découpe la narration en séquences et sépare complètement les passages narrativo-descriptifs et les dialogues, par l'usage du présent qui capte le monde fictif comme une série d'instantanés et par la simplicité d'un style dépouillé de toute marque apparente de subjectivité.

Ce qui distingue ces ciné-romans des autres scénarios, c'est leur rapport privilégié au cinéaste écrivain. À la différence des autres films, Le Souffle au cour et $A u$ revoir les enfants n'ont pas été écrits en collaboration. Ils se caractérisent par leur rapport étroit à la vie personnelle et, en particulier, à l'enfance de Louis Malle. "Le Souffle au cour, a-t-il déclaré, est lié à des images folles et imaginaires de ma propre enfance. J'ai commencé à rédiger des notes sur ce qui s'était passé quand j'ai eu mon souffle au cour " (cité dans French, p. 107). À propos d'Au revoir les enfants, le cinéaste a expliqué qu'il s'agissait du souvenir le plus dramatique de son enfance. Il raconte: "En 1944, j'avais 11 ans et j'étais pensionnaire dans un collège catholique près de Fontainebleau. L'un de mes camarades m'intriguait beaucoup [...]. J'ai commencé à le connaître, à l'aimer quand un matin, notre petit monde s'est écroulé» (cité dans Decock, p. 675).

Il ne s'agit évidemment pas d'interpréter ces ciné-romans ou, a fortiori, ces films comme des œuvres purement autobiographiques. 
Nous voulons seulement jeter un regard sur un certain mode d'écriture qui differe considérablement de la description traditionnelle du scénario comme épure du film dans les manuels. Le Souffle au cour et $A$ revoir les enfants révèlent une étape de la création bien distincte de la planification du tournage. Lécriture y correspond à la construction d'un monde fictif que Jean Alter qualifiait de littéraire. Bien qu'elle reflète indéniablement une vision cinématographique et une composition filmique, elle permet avant tout de donner un sens à des moments particulièrement significatifs appartenant au passé du cinéaste écrivain, auteur de son film au plein sens du terme, comme un romancier de son roman.

Fondé sur le souvenir d'une amitié enfantine, l'histoire d'Au revoir les enfants est bien connue: un collège cache trois enfants juifs qui seront finalement arrêtés par la Gestapo suite à une dénonciation: "Ce matin de 1944 a peut-être décidé de ma vocation de cinéaste. C'est ma fidélité, ma référence. J'aurais dû en faire le sujet de mon premier film, mais j'hésitais, j'attendais " (cité dans French, p. 116). Ce lien entre un imaginaire personnel et la créativité qu'on est peu accoutumé à déceler au cinéma a sans doute motivé le caractère particulier de l'écriture et de la forme de ce ciné-roman. À ce premier stade de la création, il s'agissait pour Louis Malle d'une confrontation avec lui-même, de la projection d'une scène primaire de sa mémoire, d'un conflit non résolu de son passé.

À partir de la description des images, le lecteur peut suivre le parcours d'un regard critique qui compose et organise les scènes d'un passé imaginaire en cherchant à leur donner un sens. Ainsi en est-il de la progression des séquences qui mènent inéluctablement à la dénonciation des enfants juifs par Joseph, le garçon de cuisine du collège. Celle-ci est préparée par une multitude d'indices visuels et de paroles lourdes de signification; Joseph y révèle l'amertume qui le conduira à la trahison:

Julien ne l'écoute pas. Il regarde Joseph, le garçon de cuisine, engagé dans une tractation à voix basse avec l'un des grands (Malle, 1987, p. 27).

Ils entendent des cris, voient un rassemblement près de la cuisine. Joseph est à terre au milieu d'un groupe 
d'élèves qui se moquent de lui et le font tomber chaque fois qu'il se relève (p. 70).

JOSEPH

Plus ils sont riches, plus ils sont voleurs (p. 28).

JOSEPH

Y a que moi qui trinque. C'est pas juste... (p. 111).

Louis Malle souligne cet effort de compréhension qui correspond au stade de l'écriture du scénario:

Je sais maintenant que ce qui m'est arrivé quand j'avais 11 ans en 1944, m’a traumatisé. Sans être vraiment capable de comprendre à l'époque, je me suis trouvé en face d'une situation qui était incompréhensible et inacceptable, et je l'ai réalisé comme un réflexe. Plus tard, quand j'ai essayé de l'analyser, c'est devenu de plus en plus inacceptable et incompréhensible et je crois que c'est ça qui m'a encouragé à devenir cinéaste: j'ai éprouvé le besoin d'examiner ce qui me hante, ce qui me fascine et ce qui me dérange (cité dans Decock, p. 675).

Le regard du cinéaste écrivain prend un autre sens que celui du réalisateur qui crée les images du film: ce premier spectacle mental lui permet de mieux comprendre le passé.

Ce regard sur l'enfance est lié au regard que Louis Malle jette sur un personnage particulier, son alter ego, le petit Julien. Dans les parties "action" du scénario, celui-ci joue fréquemment le rôle de focalisateur. Cependant, malgré la proximité affective entre l'auteur et le personnage, le point de vue distancié du narrateur se distingue manifestement de celui de l'enfant. Laccumulation des indices menant à la dénonciation se double d'une série de signes qui révèlent la découverte progressive par Julien de l'identité de son camarade et la naissance de leur amitié. Mais surtout, le ciné-roman est le lieu d'un examen de conscience qui atteint son moment le plus dramatique dans la séquence de l'arrestation par l'officier de la Gestapo:

Müller se met à marcher le long des pupitres en scrutant le regard des enfants.

Il se retourne, aperçoit la carte d'Europe avec ses petits drapeaux. Il va arracher les drapeaux russes et améri- 
cains. Il tourne le dos à Julien qui ne peut s'empêcher de regarder vers Bonnet, une fraction de seconde. Müller se retourne, intercepte le regard. Il traverse la classe lentement et vient se planter devant Bonnet (1987, p. 120).

Louis Malle a dit combien cette scène était proche de ses souvenirs, tout en indiquant une différence majeure dans la fiction du scénario:

C'est la scène vécue dont je me souvenais le plus précisément mais j'ai rajouté ce détail [le regard de Julien] quand j’a écrit la première mouture du scénario d'une façon complètement inconsciente. En relisant mon scénario, je me suis dit mais c'est bizarre pourquoi? Ça ne s'est pas passé comme ça $[. .$.$] et puis je$ me suis rendu compte que ça devait avoir une raison d'être, comme une façon de dire a posteriori que je m'étais senti responsable (cité dans Decock, p. 673).

Ce commentaire met en lumière tout l'engagement affectif personnel que représente ici l'écriture du scénario. Le cinéroman révèle donc, par la sélection des signifiants qui le distingue du foisonnement des images du film, l'élaboration d'une dramaturgie fondée sur une subjectivité. Celle-ci trouve son objectivation dans une expression visuelle de nature proprement cinématographique: une série de regards chargés de signification. Les deux autres ciné-romans publiés par Louis Malle, Le Souffle au cour et Milou en mai sont eux aussi intimement liés à l'enfance du cinéaste. En outre, ils se caractérisent par le regard critique et distancié que celui-ci porte sur le milieu de la bonne bourgeoisie française qui était le sien. Dans le cas des autres films, adaptés de romans, écrits en collaboration ou rédigés par un autre scénariste, les scénarios ont une forme et une fonction différentes. Ils présentent une autre facette d'une œuvre remarquablement diverse.

\section{François Truffaut et Antoine Doinel}

Appartenant à la même génération de cinéastes auteurs, férus de littérature, François Truffaut a lui aussi publié deux textes qui se distinguent manifestement des découpages techniques 
disponibles pour ses autres films dans la collection L'Avant-Scène du cinéma. L'Argent de poche, sous-titré "ciné-roman", absolument dépourvu de terminologie cinématographique, est rédigé dans une forme très libre qui trahit ses origines littéraires: "L'Argent de poche devait être le titre d'un recueil de nouvelles auquel j'ai renoncé pour en tirer un scénario de film" (Truffaut, 1976, p. 9). De lecture agréable, ces histoires d'enfants très simples sont proches du scénario par leur focalisation et leur organisation, mais Truffaut n'y spécifie ni cadrage, ni découpage, ni angles de caméra. Le ciné-roman ne peut évidemment communiquer le charme de l'enfance qu'ont si bien pu capter les images du film. En revanche, il révèle la fascination du metteur en scène pour le monde de l'enfance. Ainsi exprime-t-il, davantage que le film, l'engagement personnel et affectif du cinéaste écrivain. Celui-ci est présent, non pas au niveau de l'énonciation et du style, mais comme chez Louis Malle, par l'organisation des séquences, le choix des éléments signifiants qui disent l'admiration de Truffaut pour l'énergie et la vitalité des enfants. Ainsi, sa description de la dernière séquence du film où les enfants saluent par une explosion de clameurs le premier baiser de Patrick et de Martine :

Ce n'est pas un chahut hostile, ni spécialement amical; c'est un chahut tout court, une explosion de vitalité! Tous ces cris et ces rires illuminent 120 visages d'enfants, 60 garçons et 60 filles, et ces visages tous pareils et tous différents font penser à une foule chinoise, ce sont des visages d'enfants impatients de diriger leurs vies (p. 137).

L'autre ciné-roman publié par Truffaut, Les Aventures d'Antoine Doinel, contient les scénarios de cinq films où figure ce personnage privilégié de son œuvre: Les 400 coups, L'Amour à vingt ans, Baisers volés, Domicile conjugal et L'Amour en fuite. Ce livre de facture très particulière parmi les textes cinématographiques met en valeur de diverses façons la figure de l'auteur et son rapport au monde fictif. Tout d'abord, le volume contient des premières ébauches et des notes de travail documentant l'élaboration des scénarios. Bien qu'écrits en collaboration, ceuxci sont manifestement le fruit de l'imaginaire propre à ce 


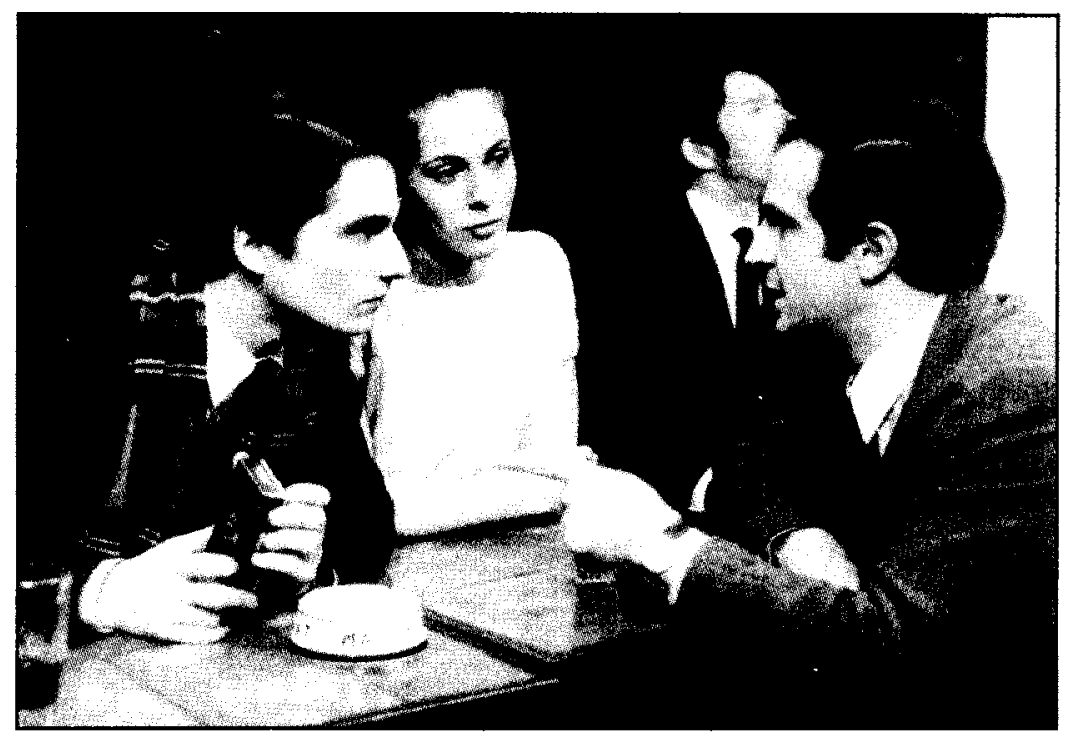

\section{Jean-Pierre Léaud, Claude Jade et François Truffaut durant le tournage de Baisers volés (1968)}

réalisateur. On peut suivre à travers ces documents, comme dans certains ciné-romans de Robbe-Grillet, la genèse du film. On y voit combien, au stade du scénario, Truffaut calcule minutieusement ses effets : "[...] nous devons établir que [...]"; "Il faut montrer que $[\ldots]$ »; "Il est possible que nous obtenions de grands avantages si $[\ldots]$ " (p. 273) et se montre particulièrement soucieux de la construction d'une dramaturgie propre à éveiller et à maintenir l'intérêt du spectateur.

Cependant, l'aspect le plus original de ce livre qui a son titre propre, différent de ceux des films, c'est de mettre en lumière la continuité entre ces quatre scénarios qui, non seulement mettent en scène le même personnage, mais racontent également des épisodes successifs de son enfance et de sa jeunesse. Le personnage d'Antoine Doinel acquiert alors, à travers cette extension de la temporalité, une véritable dimension romanesque. Le cinéroman dessine l'évolution de Doinel, de l'adolescent fugueur des 400 coups à l'homme marié du Domicile conjugal en passant par Baisers volés. Ainsi le lecteur peut-il "regarder" Antoine grandir, 
aimer et vieillir ${ }^{5}$. Les passages commentatifs des scénarios soulignent cette dimension temporelle et évolutive qui se traduit difficilement au cinéma: "Au cours du premier acte, il faut également renouer avec les beaux-parents et revenir probablement au pavillon de banlieue qui nous rappellera Baisers volés" (p. 272). Comme dans la narration romanesque, Truffaut ponctue son récit de sommaires résumant le passage des années:

Depuis la publication de son livre Les Salades de
l'amour, Antoine Doinel (Jean-Pierte Léaud) est
devenu correcteur dans une imprimerie. À cette activité
qu'il pratique depuis deux ans, Antoine ne fait qu'un
reproche, elle le détourne de l'écriture (p. 387).

Tout en regardant Antoine grandir, aimer et vieillir, Truffaut jette un regard sur son propre passé. La continuité temporelle entre les scénarios est liée à leur caractère personnel: "C’est la seule fois où j'ai su immédiatement que je ferais la suite" (p. 391). L'exploration de son passé passionnait Truffaut qui passait son temps à compléter des chronologies qu'il avait dressées de sa vie, à prendre des notes sur son enfance et ses rencontres. L'histoire des 400 coups reprend plusieurs épisodes de son adolescence. Comme dans $A u$ revoir les enfants, le scénario exprime, davantage que le film, le rapport affectif qui unit le réalisateur à son personnage. Mais cette proximité se double d'un regard ironique quand Truffaut imagine les déboires amoureux d'Antoine et qu'il se plaît à le placer dans des situations embarrassantes: «Dans le cas d'Antoine Doinel, ses mensonges m'intéressent beaucoup [...]. Je n'ai jamais vu quelqu'un montrer autant de bonne foi dans le mensonge» (p. 393).

Si le scénario fait ressortir les rapports complexes d'identification et de distanciation qui existent entre le scénariste et son personnage en arnont du film, il met aussi au jour, en aval du film, dans l'introduction et les commentaires, la dimension nouvelle apportée par Jean-Pierre Léaud. Il existe une affinité particulière entre l'acteur et le réalisateur, affinité mentale: «Mon but était de trouver une ressemblance plus morale que physique avec l'enfant que je croyais avoir été» (cité dans De Baecque). Affinité physique également. Truffaut raconte que le tenancier 
d'un bistrot lui a dit un jour: "Vous, je vous reconnais... je vous ai vu hier à la télévision. Or ce n'était évidemment pas moi que le patron avait vu à la télévision mais Jean-Pierre Léaud jouant le rôle d'Antoine Doinel" (Truffaut, 1987, p. 4). Ainsi se construit un personnage particulier au livre intitulé Les Aventures d'Antoine Doinel. Il n'a certes pas le relief de son existence à l'écran, mais sa temporalité propre et une identité ambiguë qui le distingue de l'Antoine des films: "Ce personnage imaginaire se trouve être la synthèse de deux personnes: Jean-Pierre Léaud et moi» (p. 393).

\section{Éric Rohmer, Six Contes moraux}

Comme Les Aventures d'Antoine Doinel, l'épais volume des Six Contes moraux rassemble plusieurs scénarios. En les réunissant ainsi, Rohmer fait ressortir leur thématique commune: l'angoisse ou le malaise de chaque personnage principal se trouve confronté à une sorte de cas de conscience amoureux. Dans son introduction, le réalisateur souligne ce qui distingue ces textes des découpages techniques et les rapproche, comme l'indique le titre du recueil, de la littérature: "Ces textes ne sont pas tirés de mes films. Ils les précèdent dans le temps; j'ai voulu d'emblée qu'ils fussent autre chose que des scénarios" (p. 7). Ils ont également été retravaillés en vue de publication, "pour la commodité de la lecture". Ils se caractérisent par l'absence de toute référence à une mise en scène cinématographique, tout en étant d'une forme et d'un style tout différents des novellisations.

C'est la présence d'un narrateur à la première personne qui constitue l'originalité la plus marquante de ce ciné-roman. Les premières phrases de Ma nuit chez Maud pourraient passer pour l'incipit d'une nouvelle ou d'un roman:

Je ne dirai pas tout dans cette histoire. D'ailleurs il n'y a pas d'histoire, mais une série, un choix d'événements très quelconques, de hasards, de coïncidences comme il en arrive toujours plus ou moins dans la vie, et qui n'ont d'autre sens que celui qu'il m'a plu de leur donner (p. 61).

Mais bientôt, le texte prend une forme plus proche du mode narratif du scénario: 
Le jour commence à poindre. Maud a la tête presque entièrement cachée par le drap. On distingue nettement mon visage éclairé par le reflet de l'aube. Je me réveille, je m'assois, puis je me glisse résolument sous le couvre-lit de fourrure. Maud a bougé. Elle tourne sur elle-même et se colle contre moi. Elle m'enlace. Sa main caresse mon dos. On entend nos respirations haletantes [...]. Brusquement, je me dégage et me redresse à demi (p. 98).

Le narrateur se contemple ainsi jouant un spectacle imaginaire; omniprésent, il est celui qui raconte, celui qui voit et celui qui agit dans le monde fictif. "Tout se passe, dit Rohmer, dans la tête du narrateur" (p. 10). Les ciné-romans nous présentent un jeune homme (à la fois différent et semblable dans chaque scénario) en train de se raconter son histoire comme s'il se contemplait su: un écran de cinéma. Il ne s'agit pas des commentaires en voix off qui figurent dans la partie "dialogues" des scénarios. Les curieux passages que nous avons cités sont inclus dans les parties narrativo-descriptives. La présence de ces passages est due moins à la nécessité de révéler des pensées intimes, impossibles à traduire par l'image ou par le dialogue, qu'à situer sans équivoque le point de vue du protagoniste et à faire de ce point de vue l'objet même de la visée de l'auteu. cinéaste. Centrale dans l'œuvre cinématographique de Malle, Truffaut et Rohmer, la figure de l'auteur cinéaste l'est encore bien davantage dans leurs ciné-romans. Les traits distinctifs du scénario, texte performatif préparant le tournage du film ou décrivant a posteriori son découpage technique ont disparu pour donner une importance inédire au sujet de l'énonciation, dans un discours qui présente cependant une forme indéniablement cinématographique.

\section{Ingmar Bergman : fouiller le passé}

Tous les scénarios publiés par Ingmar Bergman sont en fait des ciné-romans rédigés dans un style littéraire qui les distingue des découpages techniques ${ }^{6}$. La vision profondément personnelle qui caractérise toute son œuvre se manifeste déjà au stade de la rédaction des scénarios. Bergman y consacre de longs passages à la présentation de la vie intérieure de ses personnages. Il 
évoque à grands traits l'armosphère et le décor dans un style riche en métaphores, par des détails que la caméra ne peut enregistrer comme la personnification des objets ou la description des odeurs. En effet, ces scénarios ne préparent pas, à proprement parler, le tournage. Chez Bergman, ils créent avant tout un monde imaginaire que le réalisateur s'efforce d'évoquer pour tous ceux, acteurs et techniciens, qui vont participer à la production du film.

L'œuvre cinématographique de Bergman est l'expression d'une recherche qui, au cours de son évolution, s'oriente de plus en plus vers l'exploration d'un passé personnel. Ainsi l'écriture du scénario de Fanny et Alexandre a-t-elle représenté, pour le cinéaste écrivain, une confrontation avec lui-même et son enfance:

Progressivement, je commence à comprendre que, grâce à ce film et à un scénario qui m’oppose une résistance tenace, je tente de faire face à certains conflits personnels. Dès le début, il est évident qu'avec Fanny et Alexandre, j'ai pénétré dans le monde de ma propre enfance (Bergman, 1994, p. 366).

Cette recherche du passé, Bergman la mène par l'entremise de textes autobiographiques de forme traditionnelle: Images, ma vie en film et Laterna magica. Mais, à la toute fin de sa carrière, il recrée aussi le passé dans Les Meilleures Intentions, scénario du film et de la série télévisée réalisés par Bille August. Le scénario publié est un texte hybride, passionnant et original qui démontre toutes les possibilités littéraires du ciné-roman. Dans Les Meilleures Intentions (Den gode viljan), Bergman raconte la rencontre, les fiançailles et les premières années du mariage de ses parents, avant sa naissance. Il s'agit donc de la reconstruction d'un passé lointain, difficilement accessible, qu'il n'a pas vécu lui-même, mais que Bergman entreprend poussé par l'impérieuse nécessité de mieux se comprendre en comprenant mieux ses parents: «J'ai touché avec précaution aux visages et aux destinées de mes parents et j'ai appris pas mal de choses sur moi» (p. 11).

En dépit de la longueur (483 pages) qui l'apparente au roman, Les Meilleures Intentions présente une forme indénia- 
blement cinématographique par la séparation entre dialogues (de présentation dramatique) et parties narrativo-descriptives, par le découpage en séquences, par un mode de présentation scénique qui capte les personnages au présent, en action: "Il est absolument impossible, je pense, qu'un homme comme moi qui a toujours écrit des films, puisse simplement oublier un jour que j'écris un film. J'en ai continuellement conscience: ici, la scène atteint un sommet, et là, il faut couper [...]" (traduction du texte de Vinge, p. 286)

Mais dans le moule du scénario se coule un imaginaire qui transcende les limites coutumières à ce type de discours. Bergman fouille le passé en le projetant sur l'écran de sa mémoire. Le ciné-roman raconte la genèse et l'itinéraire de cette recherche qui débute par des rêves à propos de très anciennes photographies: « Leaning over photographs of my childhood to study my mother's face through a magnifying glass, I try to penetrate long vanished emotions» $(1988$, p. 3).

La scène commence et je prétends qu'elle commence exactement en cet instant: Anna est restée debout près du retable couvert, elle a la tête baissée, les bras ballants. Puis, elle commence à enfiler lentement ses gants qu'elle avait enlevés pour essayer l'orgue. Henrik s'avance vers la clôture du chœur, il porte un banc recouvert d'un tissu piqueté de taches et râpé. Il tourne le dos à Anna et contemple les vitres teintées du chœur. L'éclairage? Il est dramatique et très contrasté! Le soleil qui déclinait s'est arrêté devant un nuage lourd de neige surplombant la forêt (1992, p. 296).

Tout en contemplant des souvenirs inventés comme s'il s'agissait de séquences filmiques, le réalisateur souligne la part de la créativité et de l'invention: "Je ne veux pas et je ne peux pas expliquer pourquoi j'éprouve un si grand besoin de brouiller les cartes. Bon, cela peut-être fait partie du jeu - car il s'agit bien d'un jeu" (1992, p. 41).

La figure du Père dont Bergman avait peint un portrait impitoyablement sévère dans Laterna magica, son autobiographie, est au centre de son ciné-roman mais, cette fois, le rapport entre le scénariste et son personnage se transforme grâce à la 
dimension de l'imaginaire. Henrik Bergman est un homme torturé, animé des "meilleures intentions». Le ciné-roman traduit, de façon particulièrement émouvante, l'effort du metteur en scène pour se rapprocher de ces "personnages surdimensionnels, mythiques" (1992, p. 10) qui dominèrent son enfance et son adolescence. Le mode fictionnel lui permet de pénétrer dans la conscience de Henrik et de reconstruire son monde intérieur à la façon d'un romancier: "Henrik baisse les bras et le reste de l'écheveau de laine bleue retombe en même temps. Un clou s'enfonce dans sa tête. Pénètre jusqu'à sa poitrine, son ventre. Son regard se vide de toute expression» (p. 158).

Le ciné-roman présente ainsi la combinaison particulière de deux modes narratifs qui coexistent rarement dans un même texte: d'une part, l'extériorité et la visualité propres au scénario, d'autre part, l'intériorité et la présence du sujet de l'énonciation propres aux récits autobiographiques. Il va sans dire que le film imaginaire qui se crée dans l'esprit du lecteur differe considérablement des spectacles présentés au cinéma et à la télévision. Le ciné-roman n'est pas un simple sous-produit de la créativité du cinéaste écrivain. Il a sa place propre dans l'œuvre de Bergman, grâce à un style et à un mode d'écriture que celui-ci avait déjà perfectionné dans les scénarios précédents. Les Meilleures Intentions présente un exemple particulièrement accompli de l'originalité et de la lisibilité de ces nouveaux textes nés d'une rencontre entre cinéma et roman.

\section{L'écran intérieur}

Il est vrai que les textes dont nous avons parlé dans cet article different de la majorité des scénarios. Quand il est aussi le metteur en scène de son propre film, le scénariste peut produire un scénario plus personnel, non conforme aux conventions. Ces ciné-romans, dans leur particularité, mettent en relief une diversité de formes et de styles entre différents types de scripts que les manuels ont tendance à ignorer ou à condamner. On peut aussi noter les différences entre ciné-romans de cinéastes écrivains et ciné-romans d'écrivains cinéastes. Les premiers usent librement de procédés narratifs romanesques et d'un style littéraire alors que Duras et Robbe-Grillet, fascinés par la nouveauté d'une 
écriture cinématographique, en exploitent plutôt tout ce qui la distingue de la "littérature». Le trait commun prédominant entre tous ces textes, c'est, sans aucun doute, la présence de l'auteur. Ces ciné-romans portent la marque d'une forte personnalité et d'un imaginaire propre à leur créateur d'où ils tirent leur originalité et leur intérêt.

Les premières études de l'influence du cinéma sur le roman, celle de Claude-Edmonde Magny (1948) en particulier, ont mis en lumière l'avènement d'une écriture impersonnelle, orientée vers l'objectivité et l'extériorité, à l'opposé du roman d'analyse psychologique. En revanche, ces ciné-romans donnent une place centrale au sujet de l'énonciation. Chacun présente la recherche d'un temps perdu et d'un moi passé à travers le récit d'un film imaginaire. La mise en forme de cette recherche, son mode de présentation sont modelés par une vision et une écriture cinématographiques. Le ciné-roman est véritablement un texte hybride, le produit d'une fusion entre scénario et roman. Le cinéma, nul ne s'en étonnera, pénètre dans tous les domaines de l'imaginaire, y compris l'autobiographie. Dans ses mémoires personnels, Adieu, vive clarté, Jorge Semprun, romancier et scénariste, raconte ainsi sa reconstruction du passé :

\begin{abstract}
Voici le garçon de 16 ans - dans trois mois en décembre - que j'aurai été, qui m'est à la fois familier et singulièrement étranger, étrange même, comme si je contemplais une séquence cinématographique (p. 221).

Comme on revoit sa vie, semble-t-il, au moment où l'on croit mourir, ainsi pouvais-je faire défiler sur l'écran de ma mémoire le film phosphorescent de mon existence. Mais je pouvais revoir au choix n'importe quelle séquence (p. 246).
\end{abstract}

Récit d'un film imaginaire, projeté sur un écran intérieur, le scénario trouve aussi sa place dans de nombreux romans ${ }^{7}$. Presque toujours ce script fictif est présenté comme le produit de l'imagination du narrateur à la première personne. Il y projette ses fantasmes pour devenir le voyeur d'un cinéma dont la folie si débridée ne peut sans doute s'extérioriser qu'entre les pages d'un roman. Dans Le Baiser de la femme-araignée de Manuel Puig, les scénarios fictifs ont l'originalité d'être, pour la plupart, tirés de 
films réels, racontés par un spectateur et non un scénariste. Ce roman présente ainsi l'étonnante rencontre de la conscience individuelle d'un spectateur avec l'imaginaire collectif du cinéma. Molina, le narrateur, prend possession de ces films qui l'obsèdent tout en se laissant totalement investir par leur imaginaire. Il dévore le monstre cinéma tout en se laissant dévorer par lui. Ainsi scénarios fictifs et ciné-romans sont-ils le domaine privilégié de la révélation du cinéma comme projection d'une subjectivité.

\section{Simon Fraser University}

\section{Notes}

1 Voir à ce sujet Jeanne-Marie Clerc, Ecrivains et cinéma. Des mots aux images, des images aux mots. Adaptations et ciné-romans (Metz: Presses Universitaires de Metz, 1985, p. 163-210); Alain et Odette Virmaux, Un genre nouveau: Le ciné-roman (Paris: Edilig, 1983). Les premiers ciné-romans ont été publiés sous forme de feuilletons dans des quotidiens.

2 Voir la publicité pour cette collection dans "Les Écrivains cinéastes", Le Magazine littéraire, n" 354, mai 1997.

3 Voir notamment Jeanne-Marie Clerc, Écrivains et cinéma. Des mots aux images, des images aux mots. Adaptations et ciné-romans (Metz: Presses Universitaires de Metz, 1985, p. 273-342); Isabelle Raynauld, "Lire le film / voir le texte», L'Arc 98 (1985), p. 81-87; Jacqueline Viswanathan, «Les textes hybrides de Marguerite Duras: scénarios et art du non-dit", "Marguerite Duras dans les trous du discours", Cahiers de l'APFUCC (1987), p. 45-64.

4 Voir à ce sujet Jacqueline Viswanathan, «Le roman-scénario : étude d'une forme romanesque", Le Journal canadien de recherche sémiotique, vol. VII, n" 3 (1980), p. 125-151 et "L'Imaginaire du cinéma dans trois romans québécois" (Littérature et cinéma du Québec, Rome: Bulzoni Editore, 1997, p. 103-116).

5 Certaines cinémathèques ont organisé des séances de projections successives de ces films, mais ces spectacles sont évidemment l'exception.

6 Voir à ce sujet Birgitta Ingemanson, "The Screenplays of Ingmar Bergman: Personification and Olfactory Details ", Literature-Film Quarterly, vol, 12, n" 1 (1984), p. 26-33; Peter Ohlin, "Through a Glass Darkly: Figurative Language in Ingmar Bergman's Script", Scandinavian-Canadian Studies, n" 3 (1988), p. 73-88. et Louise Vinge, "The Director as Writer: some Observations on Ingmar Bergman's Den gode viljan" (dans Sarah Death et Helena Forsas-Scott (direction), A Century of Swedish Narrative. Essays in Honour of Karin Petherick, Norwich: Norvick Press, 1994, p. 281-294).

7 Voir à ce sujer Jacqueline Viswanathan, «Le roman-scénario: étude d'une forme romanesque", Le Journal canadien de recherche sémiotique, vol. VII, n" 3 (1980), p. 125-151 et "L'Imaginaire du cinéma dans trois romans québécois" (Littérature et cinéma du Québec, Rome: Bulzoni Editore, 1997, p. 103-116).

Nous avons déjà cité Prochainement sur cet écran de Pierre Turgeon (1974), Neige noire d'Hubert Aquin (1975), Histoire de caur de Jacques Savoie (1988), The Last Words of 
Dutch Schulz de William Burroughs (1975), Pilote de chasse de Kenneth Gangemi (1975). A cette liste très incomplète, ajoutons Le Désert matue de Nicole Brossard (1987), American Whiskey Bar de Michael Turner (1996), Self: a Novel de Yann Martel (1996); Reruni (1974) et Chez Charlotte and Emily (1979) de Jonathan Baumbach et bien enteridu, Le Baiser de la femme-araignée de Manuel Puig (1979).

\section{OUVRAGES CITÉS}

Alter, Jean. "Waiting for the Referent. Waiting for Godot? On Referring in Thearre", dans Anna Whiteside at Michael Issacharoff (direction), On Referring in Theatre. Indiana: Indiana University Press (1987), p. 42-56.

Aquin, Hubert. Neige noire. Montréal : La Presse, 1974.

Baumbach, Jonathan. Chez Charlotte and Emily. New York: Fictions Collective, 1979.

Baumbach, Jonathan. Reruns. New York: Fictions Collective, 1974.

Bergman, Ingmar. Images, ma vie en films. Paris: Gallimard, 1994.

Bergman, Ingmar. Les Meilleures Intentions. Paris: Gallimard, 1992.

Bergman, Ingmar. The Magic Lantern. An Autobiography. Londres: Hamish Hamilton, 1988.

Brossard, Nicole. Le Désert mauve. Montréal : L'Hexagone, 1987.

Burroughs, William. The Last Words of Dutch Schulz. New York: Viking Press, 1975.

Chiel, Deborah. Great Expectations. A Novel from the Film. New York: St. Martin, 1998.

Clerc, Jeanne-Marie. Écrivains et cinéma. Des mots aux images, des images aux mots. Adaptations et ciné-romans. Metz: Presses Universitaires de Metz, 1985.

Davis, Christopher. Philadelphia. Paris: Ramsay / Pocket Cinéma, 1994.

Baecque, Antoine de et Serge Toubiana. François Truffaut. Paris: Gallimard, 1996.

Decock, Jean. "Un cinéma du regard. Entretien avec Louis Malle». The French Review, vol. 63, n" 4 (1990), p. 671-678.

Duras, Marguerite. Le Navire Night, Césarée, Les Mains négatives, Aurélia Steiner, Aurélia Steiner. Paris: Mercure de France, 1979.

Duras, Marguerite. Détruire dit-elle. Paris: Minuit, 1969.

Duras, Marguerite. "La destruction de la parole». Cahiers du cinéma, n" 217 (1969), p. $45-57$.

French, Philip. Conversations avec Louis Malle. Paris: Denoël, 1993.

Gangemi, Kenneth. Pilote de chasse. Paris: Flammarion, 1975.

Ingemanson, Birgitta. "The Screenplays of Ingmar Bergman: Personification and Olfactory Details". Literature-Film Quarterly, vol. 12, n" 1 (1984), p. 26-33.

Janicot, Christian (direction). Anthologie du cinéma invisible. 100 scénarios pour cent ans de cinéma. Paris: Éditions Jean-Michel Place / Arte Éditions, 1995.

Magny, Claude-Edmonde. L'Âge du roman américain. Paris: Seuil, 1948.

Malle, Louis et Jean-Cliaude Carrière. Milou en mai. Paris: Gallimard, 1990.

Malle, Louis. Au revoir, les enfants. Scénario. Paris: Gallimard, 1987.

Malle, Louis. Le Souffle au caur. Scénario. Paris: Gallimard, 1971.

Martel, Yann. Self: a Novel. Toronto: Knopf Canada, 1996.

Ohlin, Peter. "Through a Glass Darkly: Figurative Language in Ingmar Bergman's Script ". Scandinavian-Canadian Studies, n 3 (1988), p. 73-88. 
Puig, Manuel. Le Baiser de la femme-araignée. Paris: Seuil, 1979.

Rohmer, Éric. Six Contes moraux. Paris: Ramsay / Poche cinéma / L'Herne, 1974.

Savoie, Jacques. Une histoire de coeur. Montréal: Boréal, 1988.

Semprun, Jorge. Adieu, vive clarté. Paris: Gallimard, 1998.

'Truffaut, François. L'Argent de poche. Ciné-roman. Paris: Flammarion, 1976.

Truffaut, François. Les Aventures d'Antoine Doinel. Paris: Ramsay / Poche cinéma / Mercure de France, 1987.

Turgeon, Pierre. Prochainement sur cet écran. Montréal : Editions du Jour, 1973.

Turner, Michael. American Whiskey Bar. Vancouver : Arsenal Pulp Press, 1997.

Vinge, Louise. "The Director as Writer: some Observations on Ingmar Bergman", dans Sarah Death et Helena Forsas-Scott (direction), A Century of Swedish Narrative. Norwich: University of East Anglia, Norvick Press (1994).

Virmaux, Alain et Odette. Un genre nouveau: le ciné-roman. Paris: Edilig, 1983.

Viswanathan, Jacqueline. "L'Imaginaire du cinéma dans trois romans québécois", Littérature et cinéma du Québec. Rome: Bulzoni Editore (1997), p. 103-116.

Viswanathan, Jacqueline. "Le roman-scénario: étude d'une forme romanesque". Le Journal canadien de recherche sémiotique, vol. VII, n"3 (1980), p. 125-151. 